\title{
SSD CONSISTENT CRITERIA AND COHERENT RISK MEASURES
}

\author{
W. Ogryczak, ${ }^{1}$ and M. Opolska-Rutkowska, ${ }^{2}$ \\ ${ }^{1}$ Warsaw University of Technology, Institute of Control \& Computation Engineering, 00-665 \\ Warsaw, Poland, ogryczak@ia.pw.edu.pl ${ }^{*},{ }^{2}$ Warsaw University of Technology, Institute of \\ Mathematics, Warsaw, Poland
}

\begin{abstract}
The mean-risk approach quantifies the problem of choice among uncertain prospects in a lucid form of only two criteria: the mean, representing the expected outcome, and the risk: a scalar measure of the variability of outcomes. The model is appealing to decision makers but it may lead to inferior conclusions. Several risk measures, however, can be combined with the mean itself into the robust optimization criteria thus generating SSD consistent performances (safety) measures. In this paper we introduce general conditions for risk measures sufficient to provide the SSD consistency of the corresponding safety measures.
\end{abstract}

keywords: decisions under risk, stochastic dominance, mean-risk.

\section{Introduction}

We consider the general problem of comparing real-valued random variables (distributions), assuming that larger outcomes are preferred. Two methods are frequently used for modeling choice among uncertain prospects: stochastic dominance, and mean-risk analysis. The former is based on an axiomatic model of risk-averse preferences but it does not provide us with a simple computational recipe. It is, actually, a multiple criteria model with a continuum of criteria. The mean-risk approach quantifies the problem in a lucid form of only two criteria: the mean, representing the expected outcome, and the risk: a scalar measure of the variability of outcomes. The mean-risk model is appealing to decision makers but it is not capable of modeling the entire gamut of risk-averse preferences. Moreover, for typical dispersion statistics used as risk measures, the mean-risk approach may lead to inferior conclusions.

\footnotetext{
*Paper written with financial support of grant 3T11C 00527 from The State Committee for Scientific Research.
}

Please use the following format when citing this chapter:

Author(s) [insert Last name, First-name initial(s)], 2006, in IFIP International Federation for Information Processing, Volume 199, System Modeling and Optimization, eds. Ceragioli F., Dontchev A., Furuta H., Marti K., Pandolfi L., (Boston: Springer), pp. [insert page numbers]. 
In this paper we analyze conditions that are necessary and sufficient for risk measures to provide the SSD consistency of the corresponding mean-risk models. Actually, we show that under simple and natural conditions on the risk measures they can be combined with the mean itself into the robust optimization criteria thus generating SSD consistent performance (safety) measures. The analysis is performed for general distributions but we also pay attention to special cases such as discrete or symmetric distributions. We demonstrate that, while considering risk measures depending only on the distributions, the conditions similar to those for the coherency are, essentially, sufficient for SSD consistency.

\section{Stochastic dominance and mean-risk models}

In the stochastic dominance approach random variables are compared by pointwise comparison of some performance functions constructed from their distribution functions. Let $X$ be a random variable representing some returns. The first performance function $F_{1}(X, r)$ is defined as the right-continuous cumulative distribution function itself: $F_{1}(X, r)=\mathbf{P}[X \leq r]$ for $r \in R$. We say that $X$ weakly dominates $Y$ under the FSD rules $\left(X \succeq_{F S D} Y\right)$, if $F_{1}(X, r) \leq F_{1}(Y, r)$ for all $r \in R$, and $X$ FSD dominates $Y\left(X \succ_{F S D} Y\right)$, if at least one strict inequality holds. Actually, the stochastic dominance is a stochastic order thus defined on distributions rather than on random variables themselves. Nevertheless, it is a common convention, that in the case of random variables $X$ and $Y$ having distributions $P_{X}$ and $P_{Y}$, the stochastic order relation $P_{X} \succeq P_{Y}$ might be viewed as a relation on random variables $X \succeq Y$ [11]. It must be emphasized, however, that the dominance relation on random variables is no longer an order as it is not antisymmetric.

The second degree stochastic dominance relation is defined with the second performance function $F_{2}(X, r)$ given by areas below the cumulative distribution function itself, i.e.: $F_{2}(X, r)=\int_{-\infty}^{r} F_{1}(X, t) d t$ for $r \in R$. Similarly to FSD, we say that $X$ weakly dominates $Y$ under the SSD rules $\left(X \succeq_{S S D} Y\right)$, if $F_{2}(X, r) \leq F_{2}(Y, r)$ for all $r \in R$, while $X$ SSD dominates $Y\left(X \succ_{S S D} Y\right)$, when at least one inequality is strict. Certainly, $X \succ_{F S D} Y$ implies $X \succ_{S S D} Y$. Function $F_{2}(X, r)$, used to define the SSD relation can also be presented as follows [12]: $F_{2}(X, r)=\mathrm{E}[\max \{r-X, 0\}]$, thus representing the mean below-target deviations from real targets.

If $X \succ_{S S D} Y$, then $X$ is preferred to $Y$ within all risk-averse preference models that prefer larger outcomes. In terms of the expected utility theory the SSD relation represent all the preferences modeled with increasing and concave utility functions. It is therefore a matter of primary importance that an approach to the comparison of random outcomes be consistent with the second 
degree stochastic dominance relation. Our paper focuses on the consistency of mean-risk approaches with the SSD.

Alternatively, the stochastic dominance order can be expressed on the inverse cumulative functions (quantile functions). Namely, for random variable $X$, one may consider the performance function $F_{-1}(X, p)$ defined as is the left-continuous inverse of the cumulative distribution function $F_{1}(X, r)$, i.e., $F_{-1}(X, p)=\inf \left\{\eta: F_{1}(X, \eta) \geq p\right\}$. Obviously, $X$ dominates $Y$ under the FSD rules $\left(X \succ_{F S D} Y\right)$, if $F_{-1}(X, p) \geq F_{-1}(Y, p)$ for all $p \in[0,1]$, where at least one strict inequality holds. Further, the second quantile function (or the so-called Absolute Lorenz Curve ALC) is defined by integrating $F_{-1}$, which provides an alternative characterization of the SSD relation,

Mean-risk approaches are based on comparing two scalar characteristics (summary statistics), the first, denoted $\mu(X)$, represents the expected outcome (reward), and the second, denoted $\varrho(X)$, is some measure of risk. The original Markowitz portfolio optimization model [9] uses the variance or the standard deviation. Several other risk measures have been later considered thus creating the entire family of mean-risk models. Risk measures in Markowitz-type meanrisk models, similar to the standard deviation, are translation invariant and risk relevant deviation type measures (dispersion parameters). Thus, they are not affected by any shift of the outcome scale $\varrho(X+a)=\varrho(X)$ for any real number $a$ and they are equal to 0 in the case of a risk-free portfolio while taking positive values for any risky portfolio. Unfortunately, such risk measures are not consistent with the stochastic dominance order [11]. Indeed, in the Markowitz model its efficient set may contain SSD inferior portfolios characterized by a small risk but also very low return [15]. Unfortunately, it is a common flaw of all Markowitz-type mean-risk models where risk is measured with some dispersion measures. In order to overcome this flaw of the Markowitz model, already Baumol [2] suggested to consider a performance measure, he called the expected gain-confidence limit criterion, $\mu(X)-\lambda \sigma(X)$ to be maximized instead of the minimization of $\sigma(X)$ itself. Similarly, Yitzhaki [18] considered maximization of the criterion $\mu(X)-\varrho(X)$ for the Gini's mean difference and he demonstrated its SSD consistency. Recently, similar consistency results have been introduced $[12,13]$ for measures corresponding to the standard semideviation and to the mean semideviation (half of the mean absolute deviation).

Hereafter, for any dispersion type risk measure $\varrho(X)$, the performance function $S(X)=\mu(X)-\varrho(X)$ will be referred to as the corresponding safety measure. Note that risk measures, we consider, are defined as translation invariant and risk relevant dispersion parameters. Hence, the corresponding safety measures are translation equivariant in the sense that any shift of the outcome scale results in an equivalent change of the safety measure value (with opposite sign as safety measures are maximized), or in other words, the safety measures distinguish (and order) various risk-free portfolios (outcomes) according to their 
values. The safety measures, we consider, are risk relevant but in the sense that the value of a safety measure for any risky portfolio is less than the value for the risk-free portfolio with the same expected returns. Moreover, when risk measure $\varrho(X)$ is a convex function of $X$, then the corresponding safety measure $S(X)$ is concave.

Relation of the SSD consistency of the safety measures directly involves criterion $\mu(X)-\varrho(X)$. However, the SSD dominance always implies the means inequality. Hence, in the case of $X \succeq_{S S D} Y$ we have both $\mu(X) \geq \mu(Y)$ and $\mu(X)-\varrho(X) \geq \mu(Y)-\varrho(Y)$. Thus, by combining inequalities, one may easily notice that $X \succ_{S S D} Y$ implies $\mu(X)-\lambda \varrho(X) \geq \mu(Y)-\lambda \varrho(Y)$ for all $0 \leq \lambda \leq 1$. On the other hand, one may just consider $\varrho_{\beta}(X)=\beta \varrho(X)$ as a basic risk measure, like the mean absolute semideviation equal to the half of the mean absolute deviation itself. In such a case one may gets another (possibly higher) upper bound for the trade-off coefficient guaranteeing the SSD consistency. Therefore, following [12], in this paper we say that the (deviation) risk measure is SSD $\alpha$-safety consistent if there exists a positive constant $\alpha$ such that for all $X$ and $Y$ :

$$
X \succeq_{S S D} Y \Rightarrow \mu(X)-\alpha \varrho(X) \geq \mu(Y)-\alpha \varrho(Y) .
$$

For the sake of simplicity, the SSD 1-safety consistency of a risk measure we will usually call simply SSD safety consistency. The relation of SSD (safety) consistency is called strong if, in addition to (1), the following holds

$$
X \succ_{S S D} Y \Rightarrow \mu(X)-\alpha \varrho(X)>\mu(Y)-\alpha \varrho(Y) .
$$

An important advantage of mean-risk approaches is that having assumed a trade-off coefficient $\lambda$ between the risk and the mean, one may directly compare real values of $\mu(X)-\lambda \varrho(X)$. If the risk measure $\varrho(X)$ is SSD $\alpha$-safety consistent, then except for random variables with identical $\mu(X)$ and $\varrho(X)$, every random variable that is maximal by $\mu(X)-\lambda \varrho(X)$ with $0<\lambda<\alpha$ is efficient under he SSD rules. In the case of strong SSD safety consistency, every such maximal random variable is, unconditionally, SSD efficient. Therefore, the strong SSD safety consistency is an important property of a risk measure.

The stochastic dominance partial orders are defined on distributions. The risk measures are commonly considered as functions of random variables. One may focus on a linear space of random variables $\mathcal{L}=L^{k}(\Omega, \mathcal{F}, \mathbf{P})$ with some $k \geq 1$ (assuming $k \geq 2$ whenever variance or any related measure is considered). Although defined for random variables, typical risk measures depend only on the corresponding distributions themselves and we focus on such measures. In other words, we assume that $\varrho(X)=\varrho(\hat{X})$ whenever random variables $X$ and $\hat{X}$ have the same distribution, i.e. $F_{1}(X, r)=F_{1}(\hat{X}, r)$ for all $r \in R$ or equivalently $F_{-1}(X, p)=F_{-1}(\hat{X}, p)$ for all $p \in[0,1]$. 
Table 1. SSD consistency limits for general distributions

\begin{tabular}{l|l|r}
\hline \multicolumn{2}{c|}{ Risk Measure } & \multicolumn{1}{c}{ Consistency } \\
\hline Standard semideviation & $\bar{\sigma}(X)$ & $1[12]$ \\
Mean absolute semideviation & $\bar{\delta}(X)$ & $1[12]$ \\
Mean absolute deviation & $\delta(X)$ & $1 / 2[12]$ \\
Conditional $\beta$-semideviation & $\Delta_{\beta}(X)$ & $1[14]$ \\
Mean abs. dev. from median & $\Delta_{0.5}(X)$ & $1[14]$ \\
Maximum semideviation & $\Delta(X)$ & $1[14]$ \\
Gini's mean difference & $\Gamma(X)$ & $1[18] \quad$ (strong [14]) \\
Tail Gini's mean difference & $\Gamma_{\beta}(X)$ & $1[14]$ \\
\hline
\end{tabular}

Table 2. SSD consistency limits for symmetric distributions

\begin{tabular}{l|r|rr}
\hline \multicolumn{2}{c|}{ Risk Measure } & \multicolumn{2}{c}{ Consistency } \\
\hline Standard semideviation & $\bar{\sigma}(X)$ & $\sqrt{2}[12]$ & (strong) \\
Standard deviation & $\sigma(X)$ & $1[12]$ & (strong) \\
Mean absolute semideviation & $\bar{\delta}(X)$ & $2[12]$ & \\
Mean absolute deviation & $\delta(X)$ & $1[12]$ & \\
Gini's mean difference & $\Gamma(X)$ & $2[14]$ & (strong) \\
\hline
\end{tabular}

Within the class of arbitrary uncertain prospects allowing to consider stochastic dominance (the class of random variables with finite expectations $\mathbf{E}\{|X|]<$ $\infty$, or $\mathrm{E}\left[X^{2}\right]<\infty$ while for standard deviation), several consistency results have been shown, as summarized in Table 1 (where the maximum value of alpha is presented). Obviously, any convex combination of measures preserves their SSD safety consistency which justifies several combined measures [8]. It turns out that when limiting the analysis to outcomes described with the symmetric distribution some consistency levels $\alpha$ increase and one gets additionally SSD 1 -safety consistency of the standard deviation (see Table 2).

\section{SSD consistency conditions}

The risk measures we consider from the perspective of the stochastic dominance are defined as (real valued) functions of distributions rather than random variables themselves. Nevertheless, in many various applications it might be more convenient to analyze their properties as functions of random variables. Recently, a class of coherent risk measures [1] have been defined by means of several axioms. The axioms depicts the most important issues in the risk comparison for economic decisions. therefore, they have been quite commonly recognized as the standard requirements for risk measures. Let us consider a linear space of random variables $\mathcal{L}=L^{k}(\Omega, \mathcal{F}, \mathbf{P})$ with some $k \geq 1$ (recall, we assume $k \geq 2$ whenever variance or any related measure is considered). A real valued performance function $C: \mathcal{L} \rightarrow \mathbf{R}$ is called a coherent risk measure on 
$\mathcal{L}$ if for any $X, Y \in \mathcal{L}$ it is monotonous ( $X \geq Y$ implies $C(X) \leq C(Y)$ ), positively homogeneous $(C(h X)=h C(X)$ for real number $h>0)$, subadditive $(C(X+Y) \leq C(X)+C(Y))$, translation equivariant $(C(X+a)=C(X)-a$, for real number $a$ ), risk relevant $(X \leq 0$ and $X \neq 0$ implies $C(X)>0$ ), where or inequalities on random variables are understood in terms 'a.s.' If $\varrho(X) \geq 0$ is a convex, positively homogeneous and translation invariant (dispersion type) risk measure, then the performance function $C(X)=\varrho(X)-\mu(X)$ does satisfy the axioms of translation equivariance, positive homogeneity, and subadditivity. Further, if $X \geq Y$, then $X=Y+(X-Y)$ and $X-Y \geq 0$. Hence, the convexity together with the expectation boundedness

$$
X \geq 0 \Rightarrow \varrho(X) \leq \mu(X)
$$

of the risk measure imply that the performance function $C(X)$ satisfies also the axioms of monotonicity and relevance [8].

In order to derive similar conditions for the SSD consistency we will use the SSD separation results. Namely, the following result [11, Th. 1.5.14] allows us to split the SSD dominance into two simpler stochastic orders: the FSD dominance and the Rotschild-Stiglitz (RS) dominance (or concave stochastic order), where the latter is the SSD dominance restricted to the case of equal means.

THEOREM 1 Let $X$ and $Y$ be random variables with $X \succeq_{S S D} Y$. Then there is a random variable $Z$ such that

$$
X \succeq_{F S D} Z \succeq_{R S} Y
$$

The above theorem allows us to separate two important properties of the SSD dominance and the corresponding requirements for the risk measures.

Corollary 2 Let $Q(X) \geq 0$ be a (dispersion type) risk measure. The measure is SSD 1-safety consistent if and only if it satisfies both the following conditions:

$$
\begin{aligned}
X \succeq_{F S D} Y & \Rightarrow \quad \mu(X)-\varrho(X) \geq \mu(Y)-\varrho(Y), \\
X \succeq_{R S} Y & \Rightarrow \quad \varrho(X) \leq \varrho(Y) .
\end{aligned}
$$

Proof. If $X \succeq_{S S D} Y$, then according to separation theorem $X \succeq_{F S D} Z \succeq_{R S} Y$ where $\mathbf{E}[Z]=\mathbf{E}[Y]$. Hence, applying (4) and (5) one gets

$$
X \succeq_{S S D} Y \Rightarrow \mu(X)-\varrho(X) \geq \mu(Z)-\varrho(Z) \geq \mu(Y)-\varrho(Y) .
$$

On the other hand, both the requirements are obviously necessary.

For strict relation $X \succ_{S S D} Y$, the separating $Z$ satisfies $X \succ_{F S D} Z$ or $Z \succ_{R S} Y$. Hence, the corresponding strong forms of both (4) and (5) are necessary and sufficient for the strong SSD 1-safety consistency. 
Condition (4) represents the stochastic monotonicity and it may be replaced with more standard monotonicity requirement

$$
X \geq Y \quad \Rightarrow \quad \mu(X)-\varrho(X) \geq \mu(Y)-\varrho(Y),
$$

where the inequality $X \geq Y$ is to be viewed in the sense o holding almost surely (a.s.). Essentially, $X \geq Y$ implies $X \succeq_{F S D} Y$, but not opposite. However, the relation $X \succeq_{F S D} Y$ is equivalent [11, Th. 1.2.4] to the existence of a probability space and random variables $\hat{X}$ and $\hat{Y}$ on it with the distribution functions the same as $X$ and $Y$, respectively, such that $\hat{X} \geq \hat{Y}$. Hence, for risk measures depending only on distributions, we consider, one gets requirements (4) and (6) equivalent. Note that for any $X \geq 0$ and $a \in R$ one gets $X+a \geq a$ while $\varrho(X+a)=\varrho(X)$ and, therefore, the monotonicity (6) implies $\varrho(X) \leq \mu(X)$. This justifies the expectation boundedness (3) as a necessary for monotonicity (6) or (4).

Condition (5) represents the required convexity properties to model diversification advantages. Note that the second cumulative distribution functions $F_{2}(X, r)$ are convex with respect to random variables $X$ [13]. Hence, taking two random variables $Y^{\prime}$ and $Y^{\prime \prime}$ both with the same distribution as $X$ one gets $F_{2}\left(\alpha Y^{\prime}+(1-\alpha) Y^{\prime \prime}, r\right) \leq F_{2}(X, r)$ for any $0 \leq \alpha \leq 1$ and any $r \in \mathbf{R}$. Thus, $\alpha Y^{\prime}+(1-\alpha) Y^{\prime \prime} \succeq_{R S} X$ and convexity of $\varrho(X)$ is necessary to meet the requirement (5).

The concept of separation risk measures properties following Theorem 1 is applicable while considering general (arbitrary) distributions. It may be, however, adjusted to some specific classes of distribution. In particular, we will show that it remains valid for a class of symmetric distributions. Indeed, a more subtle construction

$$
F_{1}(Z, r)= \begin{cases}F_{1}(X, r), & r<\mu(Y) \\ F_{1}(X, r+2(\mu(X)-\mu(Y))), & r \geq \mu(Y)\end{cases}
$$

preserves symmetry of the distribution thus leading us to the following assertion. For any symmetric random variables with $X \succeq_{S S D} Y$, there is a symmetric random variable $Z$ such that $X \succeq_{F S D} Z \succeq_{R S} Y$. One may also notice that the 'a.s.' characteristic of the FSD relation may be, respectively, enhanced for symmetric distributions.

It follows from the majorization theory $[6,10]$ that in the case of simple lotteries constructed as random variables corresponding $n$-dimensional real vectors (probability $1 / n$ is assigned to each coordinate if they are different, while probability $k / n$ is assigned to the value of $k$ coinciding coordinates) a convex, positively homogeneous and translation invariant (dispersion type) risk measure is SSD 1-safety consistent if and only if it is additionally expectation bounded (3). We will demonstrate this for more general space of lotteries. Hereafter, a lottery is a discrete random variable with a finite number of steps. 
Lemma 3 Lotteries $X$ and $Y$ satisfies $X \succeq_{R S} Y$ if and only if $F_{-2}(X, p) \geq$ $F_{-2}(Y, p)$ for all $p$ - cumulative probability of a step of $F_{1}(X, \alpha)$ or $F_{1}(Y, \alpha)$ and $F_{-2}(X, 1)=F_{-2}(Y, 1)$.

Proof. From quantile characterization of SSD we have inequality for all $p \in(0,1)$. On the other hand, we can see that $p$ - cumulative probability of steps are sufficient. Let $p_{1}, p_{2}, \ldots, p_{m}$ - cumulative probability of the steps of $F_{1}(X, \alpha)$ or $F_{1}(Y, \alpha)$, and let $c \in\left(p_{i}, p_{i+1}\right)$. Then, $F_{-2}(X, c)=$ $F_{-2}\left(X, p_{i}\right)+\left(c-p_{i}\right) F_{-1}\left(X, p_{i+1}\right)=F_{-2}\left(X, p_{i+1}\right)-\left(p_{i+1}-c\right) F_{-1}\left(X, p_{i+1}\right)$. Hence, $F_{-2}(X, c) \geq F_{-2}(Y, c)$ whenever $F_{-2}\left(X, p_{i}\right) \geq F_{-2}\left(Y, p_{i}\right)$ and $F_{-2}\left(X, p_{i+1}\right) \geq F_{-2}\left(Y, p_{i+1}\right)$. Furthermore, $F_{-2}(X, 1)=\mathrm{E}[X]=\mathrm{E}[Y]=$ $F_{-2}(Y, 1)$ is necessary for RS-dominance.

Let $X=Y_{n} \succ_{R S} Y_{n-1} \succ_{R S} \ldots \succ_{R S} Y_{1}=Y$ and for all $k: Y_{k}=$ $\lambda_{k-1} Y_{k-1}^{\prime}+\left(1-\lambda_{k-1}\right) Y_{k-1}^{\prime \prime}$, where $\lambda_{k-1} \in(0,1)$ and $Y_{k-1}^{\prime} \neq Y_{k-1}^{\prime \prime}$ are the same distributed as $Y_{k-1}$, then it is obvious to say that $Y$ is more risky that $X$ for all $\rho$ - convex risk measures. Rothschild and Stiglitz have formulated that RS-dominance between two random variables is equivalent to existing a sequence of mean preserving spreads (MPS) that transform one variable to the other. Two variables $X$ and $Y$ differ by MPS if there exists some interval that the distribution of $X$ one gets from the distribution of $Y$ by removing some of the mass from inside the interval and moving it to some place outside this interval. Gaining by MPS we will show that for lotteries with rational probability $X$ and $Y$ if only $X \succeq_{R S} Y$ then $Y$ is always more risky than $X$ for all convex risk measures.

Theorem 4 Proof. Let $X, Y$ - lotteries with rational probability of steps. If $X \succ_{R S} Y$ then there exists a sequence of lotteries $Y_{1}, Y_{2}, \ldots, Y_{n}$ satisfying the following conditions:

$$
\begin{aligned}
& 1 X=Y_{n} \succ_{R S} Y_{n-1} \succ_{R S} \ldots \succ_{R S} Y_{1}=Y \\
& 2 Y^{i+1}=\left(1-\lambda^{i}\right) Y_{1}^{i}+\lambda^{i} Y_{2}^{i} \text {, for } i=1, \ldots, n-1, \text { where } 0<\lambda^{i}<1 \text { and } \\
& Y_{1}^{i} \neq Y_{2}^{i} \text { are identically distributed as } Y^{i} .
\end{aligned}
$$

We will construct a sequence of MPS $-Y_{1}, Y_{2}, \ldots, Y_{n}$ using quantile characterization of RS-dominance. From Rothschild and Stiglitz theorem [7] one knows that the sequence $\left(Y_{k}\right)_{k=1, \ldots, n}$ exists. We will build it, however, as a convex combination of two identically distributed random variables.

Let $c_{1}, c_{2}, \ldots, c_{m}$-cumulative probability of steps of $F_{1}(X, \alpha)$ or $F_{1}(Y, \alpha)$. Due to Lemma 3 , we may focus on the steps of distributions.

Let $p_{i}=c_{i}-c_{i-1}$, for $i=2, \ldots, m$ and $p_{1}=c_{1}, \vec{p}=\left(p_{1}, \ldots, p_{m}\right)$, $x_{i}-c_{i}$-quantile of $\mathrm{X}$ for $i=1, \ldots, m, \vec{X}=\left(x_{1}, \ldots, x_{m}\right)$, $y_{i}-c_{i}$-quantile of $\mathrm{Y}$ for $i=1, \ldots, m, \vec{Y}=\left(y_{1}, \ldots, y_{m}\right)$.

There exists the first index $i$ such that $x_{i} \neq y_{i}$ (actually $x_{i}>y_{i}$, due to the 
dominance) as well as there exists the last index $i$ for which $x_{i} \neq y_{i}$ (actually $x_{i}<y_{i}$, due to the equality: $F_{-2}(X, 1)=\mathbf{E}[X]=\mathbf{E}[Y]=F_{-2}(Y, 1)$ ). With no loss of generality we can assume that the first index is 1 and the last one is $m$. By definition, we get $F_{-2}\left(Y, c_{i}\right)=\sum_{j=1}^{i} p_{j} y_{j}$.

Define: $\Delta_{j}^{i}:=x_{j}-y_{j}^{i} \forall_{i=1, \ldots, n, j=1, \ldots, m}$,

First step. $\Delta_{1}^{1}>0$ and $\Delta_{k}^{1}<0$, where $k=\min \left\{i: \Delta_{i}^{1}<0\right\}$, let $\Delta^{1}=\min \left\{\Delta_{1}^{1},-\Delta_{k}^{1}\right\}$ and $p^{1}=\min \left\{p_{1}, p_{k}\right\}$

\begin{tabular}{|c|c|c|c|c|c|c|c|}
\hline$\vec{p}^{1}$ & $p_{1}$ & & $p_{2}$ & $\ldots$ & $p_{k}$, & & $\ldots$ \\
\hline$\vec{Y}^{1}$ & $y_{1}$ & & $y_{2}$ & $\ldots$ & $y_{k}$, & & $\ldots$ \\
\hline $\overrightarrow{2} 1$ & $x_{1}$ & & $x_{2}$ & $\ldots$ & $x_{k}$, & & $\ldots$ \\
\hline & $p^{1}$ & $p_{1}-p^{1}$ & $p_{2}$ & $\ldots$ & $p^{1}$, & $p_{k}-p^{1}$ & . \\
\hline & $y_{1}$ & $y_{1}$ & $y_{2}$ & $\cdots$ & $y_{k}$, & $y_{k}$ & \\
\hline
\end{tabular}

Note that $\vec{p}^{2}$ has at least one coordinate equal $0: p_{1}-p^{1}=0$ or $p_{k}-p^{1}=0$ while $\vec{Y}^{2}$ and $\vec{X}^{2}$ have at least one new the same coordinate: $x_{1}=y_{1}^{1}+\Delta^{1}$ or $x_{k}=y_{k}^{1}-\Delta^{1}$.

With a finite number of steps we can transform $y_{1}^{1}$ to $x_{1}$ or $y_{k}^{1}$ to $x_{k}$. Thus, $m$ coordinates of $Y$ can be transformed with a finite number of steps to $m$ coordinates of $X$.

The $i$-th step has the same idea: we choose first index where $\Delta_{j}^{i}$ is positive and it can be treated as $\Delta_{1}^{1}$ in first step, because for all indexes before $\Delta_{j}^{i}=0$. Then, we choose first index when $\Delta_{j}^{i}$ is negative as $\Delta_{k}^{1}$ in first step. $\Delta^{i}, p^{i}$ are formed in the same way as $\Delta^{1}, p^{1}$.

$\vec{Y}^{i}$ is built from $\vec{Y}^{i-1}$ by moving the same mass $-\Delta^{i}$ from one coordinate to another with the same probability $-p^{i}$. Let $j, k$ be these coordinates, the rest of them are the same in the vectors.

$\begin{array}{rlllll}\vec{p}^{i}: & \ldots & p^{i}, & \ldots & p^{i}, & \ldots \\ \vec{Y}^{i}: & \ldots & y_{j}^{i}, & \ldots & y_{k}^{i}, & \ldots \\ \vec{Y}^{\prime i}: & \ldots & y_{k}^{i}, & \ldots & y_{j}^{i}, & \ldots \\ \vec{Y}^{i+1}: & \ldots & y_{j}^{i}+\Delta^{i}, & \ldots & y_{k}^{i}-\Delta^{i}, & \ldots\end{array}$

$\vec{Y}_{1}^{i}$ and $\vec{Y}^{i}$ are the same distributed and $\left(1-\lambda^{i}\right) \vec{Y}_{1}^{i}+\lambda^{i} \vec{Y}^{i}=\vec{Y}^{i+1}$ where $\lambda^{i}=\Delta^{i} /\left(y_{k}^{i}-y_{j}^{i}\right)$

$X, Y^{\prime}, Y^{\prime \prime} \in(\Omega, \mathcal{F}, \mathbf{P}) ; Y^{\prime}, Y^{\prime \prime}$ - the same distributed lotteries with rational probability of steps. If $X=\lambda Y^{\prime}+(1-\lambda) Y^{\prime \prime}$, then for all convex positive functions $\varrho$ (where $\left.\varrho\left(Y^{\prime}\right)<\infty\right)$, one gets $\varrho(X)=\varrho\left(\lambda Y^{\prime}+(1-\lambda) Y^{\prime \prime}\right) \leq$ $\lambda \varrho\left(Y^{\prime}\right)+(1-\lambda) \varrho\left(Y^{\prime \prime}\right)$. Moreover, if $\varrho$ depends only on distributions, then $\varrho\left(Y^{\prime}\right)=\varrho\left(Y^{\prime \prime}\right)$. Hence, $X \succeq_{R S} Y^{\prime}$ implies $\varrho(X) \leq \varrho\left(Y^{\prime}\right)$, and $X \succ_{R S}$ $Y^{\prime}$ implies $\varrho(X)<\varrho\left(Y^{\prime}\right)$ if $\varrho$ is strictly convex on identically distributed random variables. Recall that expectation boundedness together with convexity guarantee the corresponding monotonicity with strict monotonicity properties 
for strictly expectation bounded risk measures. This leads to the following assertion.

THEOREM 5 Let us consider a linear space $\mathcal{L} \subset L^{k}(\Omega, \mathcal{F}, \mathbf{P})$ of lotteries with rational probability of steps. If risk measure $\varrho(X) \geq 0$ depending only on distributions is convex, positively homogeneous, translation invariant and expectation bounded, then the measure is SSD 1-safety consistent on $\mathcal{L}$. If $\varrho(X)$ is also strictly convex on identically distributed random variables and strictly expectation bounded (on risky r.v.), then it is strongly SSD 1-safety consistent on $\mathcal{L}$.

Note that Theorem 5 applies to the important class of distributions where one may take advantages of the LP computable risk measures [8]. It justifies then the sufficient conditions for the coherency as simultaneously sufficient for SSD (safety) consistency. The basic consistency results could be also derived for continuous distributions from the relation [3]

$$
X \succeq_{R S} Y \quad \Leftrightarrow \quad X \in \overline{\operatorname{conv}}\left\{\hat{Y}: F_{\hat{Y}}=F_{Y}\right\} .
$$

However, the strong consistency results cannot be achieved in this way.

\section{Concluding remarks}

One may specify risk dependent performance functions to transform several risk measures into SSD consistent and coherent safety measures. We have introduced convexity and expectation boundedness as necessary and sufficient conditions which allow us to justify various risk measures with respect to such coherent transformation. While focusing on the space of finite lotteries, where one may take advantages of the LP computable risk measures, it turns out that these sufficient conditions for the coherency are also sufficient for SSD consistency. Moreover, when enhanced to strict convexity (on identically distributed random variables) and strict expectation boundedness (on risky random variables) they are also sufficient for strong SSD consistency. The latter is crucial to guarantee the SSD efficiency of the corresponding safety maximization optimal solutions.

\section{References}

[1] Artzner, P., Delbaen, F., Eber, J.-M., Heath, D. (1999), Coherent measures of risk. Math. Finance, 9, 203-228.

[2] Baumol, W.J. (1964), An expected gain-confidence limit criterion for portfolio selection. Manag. Sci., 10, 174-182.

[3] Dentcheva, D., Ruszczyński, A. (2004), Convexification of stochastic ordering constraints, Compt. Res. l'Acad. Bulgare Sci., 57, 11-16.

[4] Fishburn, P.C. (1964), Decision and Value Theory, Wiley, New York. 
[5] Hanoch, G., Levy, H. (1969), The efficiency analysis of choices involving risk, Revue Econ. Studies, 36, 335-346.

[6] Hardy, G.H., Littlewood, J.E., Polya, G. (1934), Inequalities, Cambridge University Press, Cambridge, MA.

[7] Leshno, M., Levy, H., Spector, Y. (1997), A Comment on Rothschild and Stiglitz's "Increasing risk: I. A definition", J. Econ. Theory, 77, 223-228.

[8] Mansini, R., Ogryczak, W., Speranza, M.G. (2003), LP solvable models for portfolio optimization: A classification and computational comparison, IMA J. Manag. Math., 14, $187-220$.

[9] Markowitz, H.M. (1952), Portfolio selection, J. Finance, 7, 77-91.

[10] Marshall, A.W., Olkin, I. (1979), Inequalities: Theory of Majorization and Its Applications, Academic Press, NY, 1979.

[11] Müller, A., Stoyan, D. (2002), Comparison Methods for Stochastic Models and Risks, Wiley, New York.

[12] Ogryczak, W., Ruszczyński, A. (1999), From stochastic dominance to mean-risk models: Semideviations as risk measures, European J. Opnl. Res., 116, 33-50.

[13] Ogryczak, W., Ruszczyński, A. (2001), On stochastic dominance and mean-semideviation models. Mathematical Programming, 89, 217-232.

[14] Ogryczak, W., Ruszczyński, A. (2002), Dual stochastic dominance and related mean-risk models, SIAM J. Optimization, 13, 60-78.

[15] Porter, R.B. (1974), Semivariance and stochastic dominance: A comparison, American Econ. Review, 64, 200-204.

[16] Rockafellar, R.T., Uryasev, S. (2000), Optimization of conditional value-at-risk. J. Risk, 2, 21-41.

[17] Rothschild, M., Stiglitz, J.E. (1970), Increasing risk: I. A definition, J. Econ. Theory, 2, 225-243.

[18] Yitzhaki, S. (1982), Stochastic dominance, mean variance, and Gini's mean difference, American Econ. Review, 72, 178-185. 